\title{
Educação permanente em saúde: concepções e práticas de enfermeiros de unidades básicas de saúde
}

Continuing health care education: concepts and practices of nurses in basic health units

Capacitación permanente en salud: concepciones y prácticas de enfermeros de unidades básicas de salud

Priscila Orlandi Barth ${ }^{1}$, Marines Aires ${ }^{2}$, José Luis Guedes dos Santos ${ }^{3}$, Flávia Regina Souza Ramos ${ }^{4}$

${ }^{1}$ Enfermeira, Mestre em Enfermagem. Discente do Programa de Pós-Graduação em Enfermagem, nível Doutorado, da Universidade Federal de Santa Catarina (PEN/UFSC). Florianópolis, SC, Brasil. E-mail: priscilabarth@yahoo.com.br.

${ }^{2}$ Enfermeira, Mestre em Enfermagem. Discente do Programa de Pós-Graduação em Enfermagem, nível Doutorado, da Universidade Federal do Rio Grande do

Sul. Professora da Universidade Regional Integrada do Alto Uruguai e das Missões. Porto Alegre, RS, E-mail: marines@uri.edu.br.

${ }^{3}$ Enfermeiro, Mestre em Enfermagem. Discente do PEN/UFSC, nível Doutorado. Florianópolis, SC, Brasil. E-mail: joseenfermagem@gmail.com.

${ }^{4}$ Enfermeira, Doutora em Enfermagem. Professora Associada da UFSC. Florianópolis, SC, Brasil. E-mail: flaviar@ccs.ufsc.br.

\section{RESUMO}

A Educação Permanente em Saúde é uma estratégia para a construção de novas práticas em saúde e enfermagem. Este estudo objetivou conhecer as concepções e práticas de Educação Permanente em Saúde de enfermeiros da Atenção Básica dos municípios da 19a Coordenadoria Regional de Saúde do Rio Grande do Sul, Brasil. Trata-se de uma pesquisa descritiva exploratória com base qualitativa. Os dados foram coletados por meio de entrevistas semiestruturadas com 28 enfermeiros e analisadas pela Análise Temática. Os resultados mostraram que os enfermeiros têm dúvidas sobre os significados e as práticas que envolvem a Educação Permanente em Saúde, a Educação Continuada e a Educação em Saúde. A Educação Permanente em Saúde, mesmo sendo uma política pública, ainda é realizada de forma fragmentada e pouco flexível. É necessário que os profissionais tenham clareza da direcionalidade dessa política para a transformação das práticas de saúde.

Descritores: Papel do Profissional de Enfermagem; Educação em Saúde; Educação Continuada; Profissionais da Saúde; Atenção Primária.

\section{ABSTRACT}

Continuing education in health care is a strategy for constructing new health care and nursing practices. This study aimed to identify the concepts and practices of continuing education in health held by primary care nurses in the municipalities of the 19th Regional Health Coordination of Rio Grande do Sul, Brazil. It was a descriptive exploratory study of quantitative approach. Data were collected through semi-structured interviews with 28 nurses and analyzed using Thematic Analysis. The results displayed that nurses have questions about the meaning and practice involved in continuing health care education, continuing education and health care education. Although continuing health care education is a public policy, it is still carried out in a fragmented and inflexible way. Professionals must have a clear idea this policy's directionality in order for health care practices to be transformed.

Descriptors: Nurse's Role; Health Education; Continuing Education; Health Personnel; Primary Health Care.

\section{RESUMEN}

La Capacitación Permanente en Salud es una estrategia de construcción de nuevas prácticas en salud y enfermería. Se objetivó conocer las concepciones y prácticas de Capacitación Permanente en Salud de enfermeros de Atención Básica de la 19a Coordinadora Regional de Salud de Rio Grande do Sul, Brasil. Investigación descriptiva, exploratoria, con base cualitativa. Datos recolectados mediante entrevistas semiestructuradas con 28 enfermeros, analizadas por Análisis Temático. Los resultados demostraron que los enfermeros tienen dudas acerca de los significados y las prácticas que involucra la Capacitación Permanente en Salud, Educación Continua y Educación en Salud. A pesar de que la Capacitación Permanente en Salud constituye una política pública, se realiza aún de modo fragmentario y poco flexible. Es necesario que los profesionales tengan en claro la direccionalidad de tal política hacia la transformación de las prácticas de salud.

Descriptores: Rol de la Enfermera; Educación en Salud; Educación Continua; Personal de Salud; Atención Primaria de Salud. 


\section{INTRODUÇÃO}

Com as mudanças ocorridas no decorrer dos anos 1990 pela regulamentação do Sistema Único de Saúde e nos anos 2000 pela instituição da Norma Operacional Básica de Recursos Humanos (NOB-RH), o compromisso com educação dos trabalhadores da saúde passa a ser repensada e instituída como Educação Permanente em Saúde, de modo a ultrapassar tradicionais, verticais e fragmentadas, como foco exclusivo na atualização profissional.

Como parte desse processo, o Ministério da Saúde, em 2004, institui a Política Nacional de EPS (PNEPS) pela Portaria n.198/04/GM/MS, como uma proposta estratégica para a transformação das práticas de saúde e organização dos serviços, articulando as esferas de gestão, instituições formadoras e o sistema de saúde ${ }^{(1)}$. Em 2006, o governo federal institui o Pacto pela Saúde por meio da Portaria GM/MS 399/06, o qual apresenta mudanças na gestão da Educação Permanente em Saúde (EPS), assim essa sendo reestruturada em 2007 com a instituição da Portaria n.1996/07/GM/MS ${ }^{(1-2)}$.

A PNEPS torna-se uma estratégia essencial para a dinâmica de funcionamento do SUS, subsidiando a transformação das ações e consolidando-o nos serviços de saúde. Além disso, a EPS é um dos pilares para a organização da gestão democrática e para elaboração de práticas inovadoras $^{(3)}$. As ações de EPS devem, portanto, visar o coletivo e o cenário do processo de trabalho, efetivando práticas reflexivas, éticas, críticas e humanísticas.

Direciona-se a partir das necessidades da equipe de saúde e dos usuários, buscando por meio da troca dos diferentes conhecimentos e vivências a resolutividade e integralidade das ações em saúde. É apontada como perspectiva de aprendizagem no trabalho, onde os diferentes saberes e sujeitos são envolvidos no

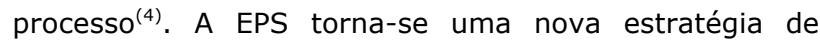
formação para a transformação das práticas de saúde com base nas necessidades sociais, não só qualificando os serviços, mas tornando o profissional mais seguro de si.

O desafio da EPS é estimular o desenvolvimento da consciência desses profissionais sobre seu contexto de atuação e sua responsabilidade no processo permanente de aprendizagem. No intuito de promover uma EPS descentralizada e interdisciplinar os enfermeiros podem desempenhar uma função estratégica, pois atuam diretamente no cuidado ao usuário/comunidade e articulam/coordenam as ações da equipe multiprofissional. Dessa forma, os enfermeiros necessitam buscar conhecimentos a partir de suas práticas, assim como estímulos, motivação e sensibilização que os levem a uma mudança de atitude em relação ao seu exercício profissional ${ }^{(5)}$.

No cenário da atenção básica, o enfermeiro possui funções complexas e se torna o profissional de referência por desenvolver inúmeras ações. Esse precisa estar presente de forma ativa e responsável em sua comunidade, assim como adotar uma nova postura nos diferentes cenários que encontra ${ }^{(6)}$. Assim, é a partir dessa mudança de agir e pensar que o enfermeiro pode ter a EPS como aliada para assegurar a qualidade de suas práticas, bem como fornecer subsídios para aplicálas com segurança e confiabilidade.

A partir dessa contextualização, justifica-se esse estudo pela necessidade de investigação da EPS no cenário da atenção básica, com enfoque no profissional enfermeiro, elo entre profissionais, comunidade, gestores e referência nos serviços de saúde.

Práticas dos enfermeiros relacionadas à implementação da EPS têm sido uma questão explorada de forma crescente na literatura científica ${ }^{(4-5,7-11)}$. No entanto, predominam estudos bibliográficos ou reflexivos sobre a EPS, com evidente lacuna de evidências científicas sobre o tema ou experiências já existentes. Além disso, pesquisas anteriores realizadas com enfoque nas ações de EPS na atenção básica e em hospitais públicos, por exemplo, evidenciaram que os profissionais ainda desenvolvem suas práticas voltadas para ações curativas, corroborando, dessa forma, a necessidade de investigações que focalize tal questão e contribua com a implementação da EPS no âmbito do SUS ${ }^{(9-12)}$.

Em um levantamento de artigos publicados entre os anos de 2006 a 2011, foram analisados 28 artigos na íntegra quanto à concepção sobre EPS, classificados em: 1. EPS como ferramenta para administração em saúde (com três artigos); 2. EPS para qualificação profissional (com 10 artigos); 3. EPS para transformação das ações em saúde (com 15 artigos). Mostra-se com esse estudo que, também pelo caráter recente desta politica, há uma diversidade de concepções, algumas vezes conflitantes com os princípios atuais, e a necessidade de se conhecer o que os enfermeiros concebem e fazem quando se referem à $\operatorname{EPS}^{(13)}$.

Desse modo, delineou-se este estudo com o objetivo de analisar as concepções e práticas relacionadas à EPS de enfermeiros que atuam na Atenção Básica.

\section{TRAJETÓRIA METODOLÓGICA}

Trata-se de um estudo descritivo-exploratório com 
abordagem qualitativa, que focaliza o contexto subjetivo dos atores sociais, dos fatos, dos conflitos, enfatizando os significados gerados pela integração social ${ }^{(14)}$.

A pesquisa foi realizada na $19^{a}$ Coordenadoria Regional de Saúde (CRS) localizada no município de Frederico Westphalen, região norte do Rio Grande do Sul (RS), que abrange 26 municípios, onde 90 profissionais enfermeiros atuam na Atenção Básica. Participaram da pesquisa 28 desses enfermeiros, que atenderam aos critérios de inclusão: atuar no mínimo há seis meses na atenção básica e interesse/disponibilidade em participar da investigação. A maioria dos participantes foram do sexo feminino, idade entre 24 e 30 anos que atuavam entre seis meses e dois anos em Estratégias de Saúde da Família.

A coleta de dados foi realizada por meio de entrevista semiestruturada no local de trabalho dos enfermeiros, entre os meses de dezembro de 2010 e janeiro de 2011. Para a realização das entrevistas, utilizaram-se duas questões norteadoras: Qual a sua concepção sobre a EPS? Quais as práticas relacionadas à EPS que você realiza no seu cotidiano de trabalho?

As entrevistas foram gravadas em um dispositivo eletrônico de áudio, transcritas na íntegra e armazenadas em um banco de dados. O encerramento da coleta de dados baseou-se no pressuposto da saturação teórica, ou seja, a coleta de dados foi finalizada no momento em que as informações coletadas começaram a se repetir ${ }^{(14-15)}$.

Para análise das informações, utilizou-se a análise temática que compreendeu três etapas: pré-análise, exploração do material e tratamento dos resultados obtidos e interpretação(14). Na fase da pré-análise, ocorreu a organização das informações e a sistematização das ideias iniciais mediante a leitura flutuante dos dados, ou seja, o contato exaustivo do material, destacando elementos principais com a finalidade de identificar unidades de registro (palavraschave). Na segunda etapa, realizou-se a exploração do material que consistiu na codificação, classificação e agregação dos dados, elaboraram-se as categorias empíricas responsáveis pela especificação do tema. Na última etapa, procedeu-se o tratamento dos resultados e interpretação, fundamentada nas premissas estabelecidas pela PNEPS ${ }^{(1-2)}$. Todo o processo foi desenvolvido exclusivamente pelas pesquisadoras, utilizando-se de arquivos digitais na forma de quadros e textos analíticos, com categorizações e sínteses parciais consecutivas à cada etapa, por meio da construção de diagramas e fluxogramas temáticos.
O projeto foi aprovado pelo Comitê de Ética em Pesquisa da Universidade Regional Integrada do Alto Uruguai e das Missões (URI/Campus de Frederico Westphalen), sob no 0073.0.284.000-10. Todos os participantes da pesquisa foram esclarecidos sobre os objetivos de estudo e a metodologia proposta e assinaram o Termo de Consentimento Livre e Esclarecido. Os princípios éticos foram respeitados procurando proteger os direitos das pessoas envolvidas, conforme preconizava, à época, a Resolução 196/96, mas com posterior verificação de plena aderência à nova Resolução (466/2012) do Conselho Nacional de Saúde ${ }^{(16)}$. O anonimato dos enfermeiros participantes do estudo foi preservado por meio da adoção de códigos para identificação dos seus depoimentos compostos pela inicial da sua categoria profissional e números atribuídos conforme a realização das entrevistas (Enf1, Enf2,..., Enf28).

\section{RESULTADOS}

Considerando os objetivos propostos, elaboraram-se duas categorias principais, "Concepções dos enfermeiros sobre a educação permanente em saúde" e "Práticas de educação permanente em saúde dos enfermeiros", formada pelas subcategorias representadas na Figura 1.

A categoria Concepções dos Enfermeiros sobre Educação Permanente em Saúde foi dividida em três subcategorias:

1) necessidade técnica científica de aprimoramento profissional;

2) capacitação profissional da equipe de saúde visando melhorar $o$ atendimento das necessidades da população/comunidade;

3) educação em/para a saúde com a comunidade.

A primeira subcategoria, recorrente em 15 depoimentos, associa o conceito de EPS ao de EC, destacando-a como a busca constante de atualização de novos conhecimentos e fazeres necessários para o exercício profissional, como pode ser constado nos depoimentos abaixo:

Pra mim educação continuada é estar em contato com o novo. Estar recebendo informações e instruções seria não parar (Enf13).

Pra mim significa estar sempre aprendendo, ou se informando, sempre estar atualizado nos assuntos, sempre estar se educando (Enf17).

É uma atualização de todos os estudos sempre, que todo mês, todo dia sempre tem alguma novidade, é estar sempre reforçando aquilo já aprendido, estar sempre estudando e se atualizando (Enf24). 
Figura 1: Diagrama das categorias principais e suas subcategorias. Frederico Westphalen, RS, Brasil, 2011.

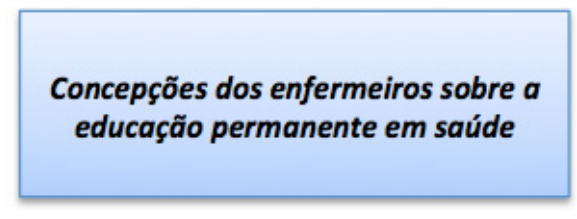

1) Necessidade técnica científica de aprimoramento profissional

2) Capacitação profissional da equipe de saúde visando melhorar o atendimento das necessidades da população/comunidade

3) Educação em/para a saúde com a comunidade

A segunda subcategoria foi a que mais se aproximou do conceito de EPS. Os participantes retrataram uma educação direcionada para as necessidades locais, pela troca de conhecimentos entre os diversos profissionais, a troca de saberes entre esses, enquanto que acena para a importância das práticas de EPS na melhoria do cuidado, presente em nove depoimentos, conforme exemplificada:

Educação permanente seria o nosso dia-a-dia, entender a nossa realidade e trabalhar em cima dos casos que se apresentam na nossa realidade, sempre surge um caso novo, algo novo para se trabalhar a educação permanente (Enf 12).

Educação Permanente seria assim, uma revisão de rotina, um acrescentar de informações periodicamente com a equipe toda, sempre estar buscando melhorar a qualidade no atendimento da população (Enf 19).

Na terceira subcategoria, os enfermeiros associaram o conceito de EPS às ações de educação em/para a saúde, numa relação direta com a clínica, o controle e a prevenção da doença, numa tradicional referencia à educação como instrumento, meio para se chegar aos fins das ações clínicas, o que esteve presente em quatro depoimentos, como nos exemplos a seguir:

A educação permanente em saúde, na verdade, eu entendo como uma prevenção (Enf4).

Educação permanente é passar orientações seja para um grupo de hipertensos ou diabéticos ou um grupo de gestantes, ou até mesmo para ACS, ou nas escolas, passando informações, dando palestras, assuntos, assim que tu vais dar uma continuidade (Enf6). Educação Permanente é assim no momento em que você começa um trabalho, principalmente na área da saúde pública, tu tem que ter com toda a população, por meio de orientações, que instruam as pessoas a se prevenir das patologias (Enf26).

$\mathrm{Na}$ categoria principal "Práticas de Educação Permanente em Saúde dos Enfermeiros", os participantes relataram tanto atividades de EPS da qual eles participavam (dirigida aos enfermeiros), quanto ações que eles realizavam com a equipe de saúde e os usuários.

Em relação às ações de EPS das quais os enfermeiros participavam, evidenciou-se que eles procuravam realizar cursos/treinamentos oferecidos pela secretaria ou CRS para se aperfeiçoar, como também buscavam por cursos de Pós-Graduação:

A única coisa que nós discutimos na verdade é quando vem das capacitações, algumas nós discutimos entre nós que somos as enfermeiras [...] (Enf 28).

Os cursos que nós temos, que são fornecidos pela 19a, pelo posto de saúde também, acho que isso também é educação, cada mês ou a cada quinze dias aparece uma capacitação para nós (Enf 22).

Nós temos um grupo grande de enfermeiros, então nós estamos agora fazendo uma pós em gestão da saúde (Enf 1)

Quanto às ações de EPS realizadas pelos enfermeiros, constatou-se que eles possuíam três focos principais: a equipe de enfermagem e saúde, os Agentes Comunitários de Saúde (ACS) e os usuários/comunidade, conforme os depoimentos abaixo:

Nós fazemos reuniões semanalmente com as ACS e com as técnicas, [...] não marcamos nenhuma, eu sento com elas informalmente e discutimos o trabalho delas (Enf5). [...] nós fazemos com o ACS de quinze em quinze dias 
(Enf 6).

Eu direciono assim, depende da área, da questão principal, posso ter acesso a gravidez na adolescência, a métodos contraceptivos, posso ter um outro bairro, uma outra área que eu não sei se é isso que precisa, então é difícil, tem que ver o momento (Enf 10).

Eu trabalho educação permanente relacionada com as agentes de saúde (Enf 18).

Aqui a educação permanente é realizada com dinâmicas, eu procuro assuntos atualizados, até material em Power Point, essas coisas, assim eu proporciono para a equipe (Enf 22).

A minha prática acaba sendo mais com os pacientes em si, de estar sempre revendo se eles estão tomando os medicamentos corretos, de estar sempre explicando a forma de tomar o remédio correto, de estar sempre dando informações acerca dos medicamentos, da própria patologia deles (Enf 24).

\section{DISCUSSÃO}

Na categoria Concepções dos enfermeiros sobre EPS, a primeira subcategoria "Necessidade técnica cientifica de aprimoramento profissional" expressa a concepção de EPS centrada no "recebimento" de conhecimentos, não sendo realizada de forma participativa, mas como busca pessoal ou oferta de oportunidades de aperfeiçoamento, identificadas por alguns autores como educação continuada (EC). Embora não se possa limitar a EC a algumas formas tradicionais, ela é por vezes referida como sendo realizada em forma de cursos, capacitações e atualizações, de maneira verticalizada, ou seja, não parte da necessidade do profissional, mas sim de conteúdos pré-estabelecidos que visem um conhecimento apenas técnico ${ }^{(8)}$.

A busca contínua por novos conhecimentos é uma condição para a inserção e manutenção dos profissionais no mercado de trabalho, que se apresenta também na área da saúde e enfermagem. No entanto, a EPS e EC são formas distintas de se estabelecer uma educação no trabalho. Enquanto a EC é centrada na transmissão de saberes, em cursos, e realizada de forma fragmentada, a EPS busca a valorização do trabalho, a participação dos profissionais no processo de ensino aprendizagem e a transformação de suas práticas ${ }^{(9)}$.

Apesar dessa diferença, autores ${ }^{(17)}$ já consideram que a educação continuada também deve ser realizada visando às necessidades que emergem a partir do cotidiano dos profissionais e da instituição de saúde. Desse modo, apesar de possuírem conceptualizações distintas, ambas possuem uma mesma direcionalidade, efetuar a educação no serviço para que essa seja ferramenta transformadora das ações em saúde.

A segunda subcategoria "Capacitação profissional da equipe de saúde visando melhorar o atendimento das necessidades da população/comunidade" expressa a percepção de EPS que se aproxima dos princípios estabelecidos pela PNEPS, pois os profissionais se pautam na troca de conhecimentos entre os diversos saberes e a busca por atualizações a partir da realidade de cada população. A PNEPS expressa que as ações de saúde devem ser direcionadas para as reais necessidades da população, usando-se da problematização para efetivar o processo de ensino aprendizagem no trabalho(10). Essa problematização não está clara nas falas dos profissionais, mas é percebida no momento em que esses utilizam das necessidades da comunidade para iniciarem e desenvolverem seu processo de EPS.

A EPS utiliza-se da integração entre realidade, problematização e educação no trabalho, enfatizando a ampliação das relações entre profissionais e usuários, para que se possa efetivar um atendimento integral e humanizado. Diante disso, a EPS ultrapassa o lócus de aprendizagem individual, da transmissão de saberes e das relações de fragmentação entre categorias profissionais, visando à interdisciplinaridade nos serviços de saúde.

A terceira subcategoria "Educação em/para a saúde com a comunidade" esboça a percepção da EPS com os conceitos de educação em/para saúde, a qual visa à qualidade de vida da população, a promoção da saúde e a troca de saberes entre profissionais e comunidade, despertando a população para a criticidade de suas ações em saúde ${ }^{(18)}$. Enquanto a EPS possui sua direcionalidade para o profissional, a educação em saúde tem o usuário como elemento chave, essa distinção inicial evidencia a falta de clareza que os enfermeiros possuem sobre EPS.

A PNEPS parte do desenvolvimento da educação no trabalho, a partir da realidade dos profissionais, em que o processo de trabalho é reflexivo e participativo, fortalecendo o crescimento e adesão do SUS ${ }^{(3)}$. Assim como nos achados deste estudo, pesquisa anterior desenvolvida com enfermeiros da atenção básica também identificou o desconhecimento desse profissional sobre a PNEPS e os conceitos descritos na literatura(19). Isso reflete na necessidade de discussão da temática nos serviços de saúde, para que essa possa ser implementada de maneira efetiva, como ferramenta estratégica para a transformação do SUS. 
Para efetivar a EPS, é necessário buscar o conhecimento a partir das necessidades do cotidiano de trabalho dos profissionais, do mesmo modo que a educação em saúde, sendo construída por meio da problematização da realidade local. A educação em saúde deve ter papel potencializador para romper e contribuir na subjetividade dos sujeitos, o compartilhamento de saberes, a autonomia, o diálogo, as relações mais igualitárias, e a criticidade, devem estar entremeadas nesse processo ${ }^{(20)}$.

A articulação entre educação e saúde é um desafio que envolve interfaces de diversos saberes, há a necessidade do diálogo entre as disciplinas e alternativas metodológicas, para que assim gere mudança de saberes e práticas na saúde ${ }^{(21)}$. Essa articulação é imprescindível para que possam ser realizadas ações efetivas de EPS, que requerem a articulação entre ensino, serviço, gestão e controle social.

A segunda categoria principal "Práticas de Educação Permanente em Saúde dos Enfermeiros" expressa práticas de EPS realizadas para e por esses. Nos depoimentos expostos nos resultados percebe-se que eles têm uma preocupação em relação a sua atualização profissional e que existe a consciência que a educação é um processo permanente. Entretanto, está implícito nas falas que as capacitações propostas pela secretaria ou CRS são vistas como uma "obrigação", direcionadas especificamente para as funções que eles exercem nos serviços de saúde, e as discussões geradas a partir das capacitações ficam, muitas vezes, circunscritas a uma categoria profissional.

É necessário que o enfermeiro rompa o paradigma de ensino-aprendizagem tradicional por meio do estabelecimento da educação no trabalho, buscando a sua inserção em um espaço onde o aprender é inacabado, se dá a todo instante e em qualquer cenário. Nessa linha de pensamento, a EPS propõe que os profissionais assumam seus papéis de coautores de suas ações, que busquem a modificação de suas práticas e que em seu coletivo produzam novos modos de se gerir e agir em saúde ${ }^{(11)}$.

Algumas dificuldades como a falta de recursos humanos e materiais, a sobrecarga de trabalho e a falta de motivação das chefias em desenvolver a educação continuada nos serviços, tornam-se agravantes para a anuência dos profissionais nos processos de capacitações $^{(17)}$. Esses entraves devem ser amenizados no ambiente de trabalho, proporcionando ao trabalhador sua participação nos processos de EP, pensando em uma educação direcionada ao trabalho, às necessidades cotidianas e a resolutividade dos problemas.

Nesse cenário, destacam-se as práticas de EPS de forma pontual e relacionadas a assuntos específicos, estando limitada às reuniões de equipe e à atualização em torno de demandas técnicas. Essas ações são importantes para o preparo técnico dos profissionais de saúde e contribuem para a segurança do paciente e qualidade do cuidado. No entanto, é preciso é ir além da dimensão técnica e também discutir, refletir e analisar as incongruências presentes no cotidiano do processo de trabalho.

A PNEPS destaca que a atualização técnico-científica passa a ser complementar ao processo de EPS dos profissionais, onde a formação e o desenvolvimento englobam aspectos da subjetividade, de habilidades técnicas e de conhecimento do SUS ${ }^{(1)}$. Para tanto, a EPS deve ser realizada de maneira interdisciplinar, ascendente, compartilhada, por meio da adoção de metodologias que transpassem a dimensão técnicocientífica e abordem a aprendizagem significativa, vislumbrando um atendimento integral, humanizado e resolutivo.

Em relação às ações de EPS desenvolvidas pelos enfermeiros e voltadas para os ACS, destaca-se a dimensão gerencial do trabalho do enfermeiro. Os participantes do estudo referiram a responsabilidade da capacitação dos ACS.

Nas entrevistas, destaca-se a ênfase da EPS para os ACS e uma ação de EPS com as técnicas de enfermagem é relatada, sem considerá-la uma ação de EPS. Dessa feita, vale lembrar que a EPS é aprendizagem no trabalho, que se dá por meio das relações do cotidiano dos profissionais e das organizações ${ }^{(2)}$. No que tange às práticas de EPS para os ACS, constata-se o quanto o enfermeiro assumiu para si a responsabilidade pela qualificação dos ACS, estabelecendo uma relação de transmissão de saberes para esses profissionais e não uma relação de troca.

A partir da implantação da Política Nacional da Atenção Básica por meio da Portaria 648/06 e sua ampliação pela Portaria 2.488/11, é expresso nesse documento que uma das atividades do enfermeiro na atenção básica é a de planejar, gerenciar e avaliar as ações desenvolvidas pelos ACS em conjunto com outros membros da equipe ${ }^{(22)}$. Seguindo a proposta do Ministério da Saúde, o enfermeiro deve estar desenvolvendo atividades que contribuam para a formação do ACS, no entanto essas devem ter caráter participativo e compartilhado, buscando por meio da troca de saberes e experiências a qualidade nas práticas 
desenvolvidas por esse profissional.

Estudo com enfermeiros de uma ESF constatou que esses são responsáveis pela supervisão e liderança do trabalho realizado pelos ACS por estarem mais próximos deles no dia-a-dia de sua prática, servindo de referência. Assim, eles procuram auxiliar na identificação, análise e resolução dos problemas que lhe são apresentados(23) indo de encontro com os achados do nosso estudo. Outra pesquisa apontou que o enfermeiro desenvolve nos ACS a compreensão de toda prática e autonomia para a construção do conhecimento, no entanto, para que o processo de EPS seja efetivo, é necessária a participação e colaboração de toda a equipe ${ }^{(19)}$.

Frente a isso, salienta-se o potencial do enfermeiro no desenvolvimento de atividades de EPS não só com os ACS, mas com toda a equipe e essa atividade deve ser fundamentada nos princípios de aprendizagem no trabalho, compartilhamento de saberes e experiências, de forma descentralizada e ascendente.

Em outro contexto, alguns participantes remeteram suas práticas de EPS direcionadas à população, enfatizando-se aqui o conflito entre a EPS e educação em saúde, evidenciado na concepção de EPS desses profissionais. Infere-se que alguns profissionais estão realizando ações de educação em saúde sem realizar uma distinção entre as ações de EPS, pois ainda possuem um pensamento ambíguo sobre essas práticas.

As ações de EPS desenvolvidas pelos entrevistados são de educação em saúde, voltadas ao cuidado com o paciente, bem como uma tradicional transmissão de saberes. Além dessa relação equivocada entre EPS e educação em saúde, há uma percepção de educação em saúde caracterizada como prática de transmissão de informação, enfatizando o conhecimento técnicocientífico e não a troca de saberes e vivências.

A educação em saúde constitui-se em um espaço de troca de conhecimentos, de práticas relacionadas à cultura dos indivíduos, sendo realizada de forma convergente aos pressupostos da promoção da saúde ${ }^{(18)}$. A educação em saúde, quando efetivada a partir da promoção da saúde, se torna uma peça chave para que a EPS seja consolidada no cotidiano dos profissionais de saúde, pois a educação em saúde promove o acesso às reais necessidades da população e essas serão direcionadoras para a EPS. Para tanto, é indispensável que os profissionais saibam diferenciar estas práticas para que possam exercê-las de maneira eficaz.

Nos depoimentos apresentados, ressalta-se que as ações de educação em saúde desenvolvidas são fomentadas a partir da problematização da realidade e desse modo podem ser direcionadas para a consolidação das ações de EPS. Todo processo de educação em saúde deve ser direcionado pelo intercâmbio de saberes técnico-científicos e populares, no qual profissionais e usuários constroem de forma compartilhada o saber em saúde, por meio de ações criticas, reflexivas e participativas, que promovam a mudança de hábitos e comportamentos ${ }^{(24)}$.

É necessário que a EPS seja construída na e para a realidade local, focalizando-se na aprendizagem no trabalho. É por meio dessas condutas que a EPS consolidar-se-á como prática transformadora nas ações dos profissionais e potencializadora na efetivação da interdisciplinaridade, da gestão compartilhada, da integralização do ensino no cotidiano do trabalho e na integralidade e humanização da assistência.

\section{CONSIDERAÇÕES FINAIS}

A pesquisa ilustra que alguns enfermeiros ainda trazem consigo a carga de um modelo tradicional de educação, especialmente quando relatam formas de EPS baseadas nas premissas da educação continuada, fragmentada, hierarquizada, centrada em cursos e capacitação. A EPS busca romper com essas formas de aprendizagem no trabalho, ultrapassando barreiras hierárquicas, buscando a interdisciplinaridade, a constante troca de saberes e vivências entre os profissionais.

Evidenciou-se também a falta de conhecimento sobre a PNEPS e os conceitos de educação em saúde, tidos como sinônimos por alguns profissionais. Assim, ressalta-se a importância desses possuírem conhecimentos sobre as políticas que regem o sistema, o que se mostra de modo ainda parcial, fazendo transparecer suas dificuldades no momento de efetiválas.

A partir dos resultados obtidos, constatou-se a falta de conhecimento dos enfermeiros sobre as políticas que regem o SUS. Sendo assim, o grande desafio apreendido com este estudo é a dificuldade de modificar os serviços de saúde se os próprios profissionais não identificam sua funcionalidade. É notória a necessidade de discussão dos preceitos da EPS, assim como das políticas públicas a partir da formação profissional, se tornando um processo contínuo e inacabado. Dentro dos limites de um estudo local, observa-se uma contribuição para a análise crítica aplicável a diferentes cenários da APS.

Dessa forma, o estudo pretende contribuir para a formação e qualificação dos profissionais de saúde, ao sinalizar a importância da adoção de uma postura crítica 
e reflexiva frente aos processos educativos e formativos. É fundamental que esses profissionais estejam direcionados para o fortalecimento das políticas públicas, bem como, saibam diferenciá-las para que possam exercê-las de forma efetiva em seu cotidiano de

\section{REFERÊNCIAS}

1. Portaria N0198/GM/MS (BR). Institui a Política Nacional de Educação Permanente em Saúde como estratégia do Sistema Único de Saúde para a formação e o desenvolvimento de trabalhadores para o setor e dá outras providências. Brasília (DF) 2004 fev.

2. Portaria N01.996 GM/MS (BR). Substitui a Portaria GM/MS no 198, de 13 de fevereiro de 2004. Política Nacional de Educação Permanente em Saúde. Departamento de Gestão da Educação na Saúde. Secretaria de Gestão do Trabalho e da Educação na Saúde. Brasília (DF) 2007 ago.

3. Merhy EE, Feuerwerker LCM, Ceccim RB. Educácion Permanente en Salud: una Estrategia para Intervenir en la Micropolítica del Trabajo em Salud. Salud Coletiva. 2006;2(2):147-160.

4. Fortuna CM, et.AL. Educação permanente na estratégia saúde da família: repensando

5. Paschoal AS, Mantovani MF, Méier MJ. Percepção da educação permanente continuada e em serviço para enfermeiros de um hospital de ensino. Rev esc. enferm. USP. 2007;41(3):478-484. 6. Backes DS, et.AL. O papel profissional do enfermeiro no Sistema Único de Saúde:

7. Ceccim RB. Educação Permanente em Saúde: descentralização e disseminação da capacidade pedagógica na saúde. Cien Saude Colet. $2005 ; 10(4): 975-986$.

8. Carotta F, Kawamura D, Salazar J. Educação permanente em saúde: uma estratégia de gestão para pensar, refletir e construir práticas educativas e processos de trabalho. Saúde e Sociedade. 2009;18 Suppl 1:S48-51.

9. Peduzzi M. et.Al. Atividades educativas de trabalhadores na atenção primária: concepções de educação permanente e educação continuada em saúde presentes no cotidiano de unidades básicas de saúde em São Paulo. Interface- Comunic., Saude, Educ. 2009;13(30):121-134.

10. Murofuse NT, Rizzotto MLF, Muzzolon ABF, Nicola AL. Diagnosis of the situation of health workers and the training process at a regional center for professional health education. Rev. latino-am. enfermagem. 2009;17(3):314-320.

11. Pinto EEP et.al. Desdobramentos da Educação Permanente em Saúde no município de Vitória, Espírito Santo. Trab. Educ. e Saude. 2010;8(1):77-96.

12. Tronchin DMR et.al. Educação permanente de profissionais de saúde em instituições públicas hospitalares. Rev esc. enferm. USP. 2009;43(2):210-1215.

13. Barth PO. Educação Permanente em Saúde: Concepções e Práticas em Centros de Saúde de Florianópolis/SC [dissertação]. Florianópolis: Programa de Pós Graduação em Enfermagem/UFSC; 2012. 92p.

14. Minayo MC. Desafio do conhecimento: pesquisa qualitativa em saúde. 8 ed. São Paulo: Hucitec; 2004.

15. Polit DF, Beck CT, Hungler BP. Pesquisa em Enfermagem: Métodos, avaliação e utilização. 5 ed. Editora: Artmed; 2004. 16. Resolução n.466 de 12 de Dezembro de 2012. Conselho Nacional de Saúde, 2012.

17. Costa DB, Vannuchi MTO, Haddad MCFL, Cardoso MGP, Silva LG, Garcia SD. Custo de educação continuada para equipe de enfermagem de um hospital universitário público. Rev. Eletr. Enf. [Internet]. [cited 2012/abr/jun;14(2):257-66. Available from: http://www.fen.ufg.br/revista/v14/n2/v14n2a05.htm. 18. Colomé JS, Oliveira DLLC. A educação em saúde na perspectiva de graduandos de enfermagem. Rev Gaúcha Enferm. 2008;29(3):347-353.

19. Barbosa VBA, Ferreira MLSM, Barbosa PMK. Educação trabalho. A EPS é essencial para que os trabalhadores de saúde repensem suas ações, busquem por meio da problematização da realidade a integralidade, a resolutividade e humanização da assistência, tornandose protagonistas na consolidação do SUS. permanente em saúde: uma estratégia para a formação dos agentes comunitários de saúde. Rev Gaúcha Enferm. 2012;33(1):56-63.

20. Renovato RD, Bagnato MHS. Da educação sanitária para a educação em saúde (1980-1992): discursos e práticas. Rev. Eletr. Enf. [Internet]. 2012 jan/mar;14(1):77-85. Available from: http://www.fen.ufg.br/revista/v14/n1/v14n1a09.htm.

21. Gubert E, Prado ML. Desafios na prática pedagógica na educação profissional em enfermagem. Rev. Eletr. Enf.

[Internet]. [cited $2011 \mathrm{abr} / \mathrm{jun}$ ];13(2):285-295. Available from: http://www.fen.ufg.br/revista/v13/n2/v13n2a15.htm.

22. Política Nacional de Atenção Básica. Secretaria de Atenção à Saúde. Departamento de Atenção Básica. Série E. Legislação em Saúde. Brasília (DF) 2012.

23. Colomé ICS, Lima MADS, Davis R. Visão de enfermeiras sobre as articulações das ações de saúde entre profissionais de equipes de saúde da família. Rev esc. enferm. USP. 2008;42(2):256-261.

24. Sá LD, Gomes ALC, Carmo JB, Souza KMJ, Palha PF, Alves RS et al. Educação em saúde no controle da tuberculose: perspectiva de profissionais da estratégia Saúde da Família. Rev. Eletr. Enf. [Internet]. 2013 jan/mar;15(1):103-11. Disponível em: http://dx.doi.org/10.5216/ree.v15i1.15246.

Artigo recebido em 07/01/2013.

Aprovado para publicação em 07/01/2013.

Artigo publicado em 30/09/2014. 